Supporting Information to:

\title{
Synthesis of Novel Melilite-Type Iron/Cobalt Oxides and Their Oxygen Evolution Reaction Electrocatalytic Activity
}

Satoshi Ogawa, ${ }^{\dagger}$ Yasuyo Ogino,${ }^{\dagger}$ Masao Yonemura,${ }^{\ddagger}$ Toshiharu Fukunaga, ${ }^{\S}$

Hisao Kiuchi, ${ }^{\S}$ Kei Nakayama, Ryo Ishikawa, ${ }^{1, \nabla}$ Yuichi Ikuhara,", O

Yoshihiro Doi ${ }^{\perp}$ Kenta Suzuki, ${ }^{\dagger}$ Miwa Saito, ${ }^{\dagger}$ Teruki Motohashi ${ }^{\dagger}, *$

†Department of Materials and Life Chemistry, Kanagawa University, Yokohama, Kanagawa 221-8686, Japan

Institute of Material Structure Science, High Energy Accelerator Research Organization 203-1 Shirakata, Tokai, Ibaraki 319-1106, Japan

§office of Society-Academia Collaboration for Innovation, Kyoto University, Gokasho, Uji, Kyoto 611-0011, Japan

'Institute of Engineering Innovation, University of Tokyo, Bunkyo, Tokyo 113-8656, Japan

${ }^{\nabla}$ Japan Science and Technology Agency, PRESTO, Kawaguchi, Saitama 332-0012, Japan ONanostructures Research Laboratory, Japan Fine Ceramics Center, Atsuta, Nagoy a 4568587, Japan

${ }^{\perp}$ Division of Chemistry, Graduate School of Science, Hokkaido University, Sapporo 0600810, Japan

${ }^{*}$ Corresponding Author

t-mot@,kanagawa-u.ac.jp 


\section{Contents}

Table S1. (a) XRD patterns for $\mathrm{Sr}_{2} \mathrm{Fe}_{2}-2 x \mathrm{Co}_{x} \mathrm{Ge}_{1+x} \mathrm{O}_{7}(0 \leq x \leq 1)$. (b) The lattice constants $a$ and $c$ against $x$ in $\mathrm{Sr}_{2} \mathrm{Fe}_{2}-2 x \mathrm{Co}_{x} \mathrm{Ge}_{1+x} \mathrm{O}_{7}$.

Figure S2. XRD patterns for $\mathrm{Ba}_{2} \mathrm{Fe}_{2}-2 x \mathrm{Co}_{x} \mathrm{Ge}_{1+x} \mathrm{O}_{7}(0 \leq x \leq 1)$.

Figure S3. (a) ORR and (b) OER polarization curves of melilite-type Fe/Co oxides.

Figure S4. (a) Fe $K$-edge and (b) Co $K$-edge XANES spectra of melilite-type $\mathrm{Sr}_{2} \mathrm{Fe}_{0.67} \mathrm{Co}_{0.67} \mathrm{Ge}_{1.66} \mathrm{O}_{7}$ together with those of the reference samples.

Figure S5. Neutron powder diffraction profiles of (a) $\mathrm{Sr}_{2} \mathrm{Fe}_{2} \mathrm{GeO}_{7}$ and (b) $\mathrm{Sr}_{2} \mathrm{Fe}_{0.4} \mathrm{Co}_{0.8} \mathrm{Ge}_{1.8} \mathrm{O}_{7}$.

Table S1. Structural parameters of $\mathrm{Sr}_{2} \mathrm{Fe}_{2}-2 x \mathrm{Co}_{x} \mathrm{Ge}_{1+x} \mathrm{O}_{7}(x=0,0.67$ and 0.80$)$.

Figure S6. Tafel plots for the OER data of melilite-type $\mathrm{Sr}_{2} \mathrm{Fe}_{2}-2 x \mathrm{Co}_{x} \mathrm{Ge}_{1+x} \mathrm{O}_{7}$.

Table S2. BET specific surface areas of melilite-type $\mathrm{Sr}_{2} \mathrm{Fe}_{2-2 x} \mathrm{Co}_{x} \mathrm{Ge}_{1+x} \mathrm{O}_{7}$.

Figure S7. OER polarization curves of melilite-type $\mathrm{Sr}_{2} \mathrm{Fe}_{2}-2 x \mathrm{Co}_{x} \mathrm{Ge}_{1+x} \mathrm{O}_{7}$ together with that of the reference catalyst $\mathrm{RuO}_{2}$. The current density values are normalized by the specific surface area of the catalyst.

Experimental: synthesis of $\mathrm{Fe} / \mathrm{Co}$-mixed oxides of various structural types.

Figure S8. XRD patterns for the Fe/Co-mixed oxides of various structural types.

Figure S9. Crystal structures of (a) perovskite-type, (b) garnet-type, and (c) spineltype compounds.

Table S3. Specific surface areas of the Fe/Co compounds of various structural types.

Table S4. Chemical composition ratios of the $\mathrm{Sr}_{2} \mathrm{Fe}_{0.67} \mathrm{Co}_{0.67} \mathrm{Ge}_{1.66} \mathrm{O}_{7}$ catalysts determined by EDX analysis.

Experimental: Durability tests for the $\mathrm{Sr}_{2} \mathrm{Fe}_{0.67} \mathrm{Co}_{0.67} \mathrm{Ge}_{1.66} \mathrm{O}_{7}$ catalysts.

Figure S10. XRD patterns for (a) as-prepared $\mathrm{Sr}_{2} \mathrm{Fe}_{0.67} \mathrm{Co}_{0.67} \mathrm{Ge}_{1.66} \mathrm{O}_{7}$, (b) the sample immersed in a $4 \mathrm{M} \mathrm{KOH}$ aqueous solution, and (c) the sample electrochemicallytreated at $1.70 \mathrm{~V}$ vs RHE.

Figure S11. Chronoamperometric curve of the $\mathrm{Sr}_{2} \mathrm{Fe}_{0.67} \mathrm{Co}_{0.67} \mathrm{Ge}_{1.66} \mathrm{O}_{7}$ catalyst with an applied potential of $1.70 \mathrm{~V}$ vs RHE.

Figure S12. Chronopotentiometric curve of the $\mathrm{Sr}_{2} \mathrm{Fe}_{0.67} \mathrm{Co}_{0.67} \mathrm{Ge}_{1.66} \mathrm{O}_{7}$ catalyst with an applied current density of $10 \mathrm{~mA} / \mathrm{cm}^{2}$. 


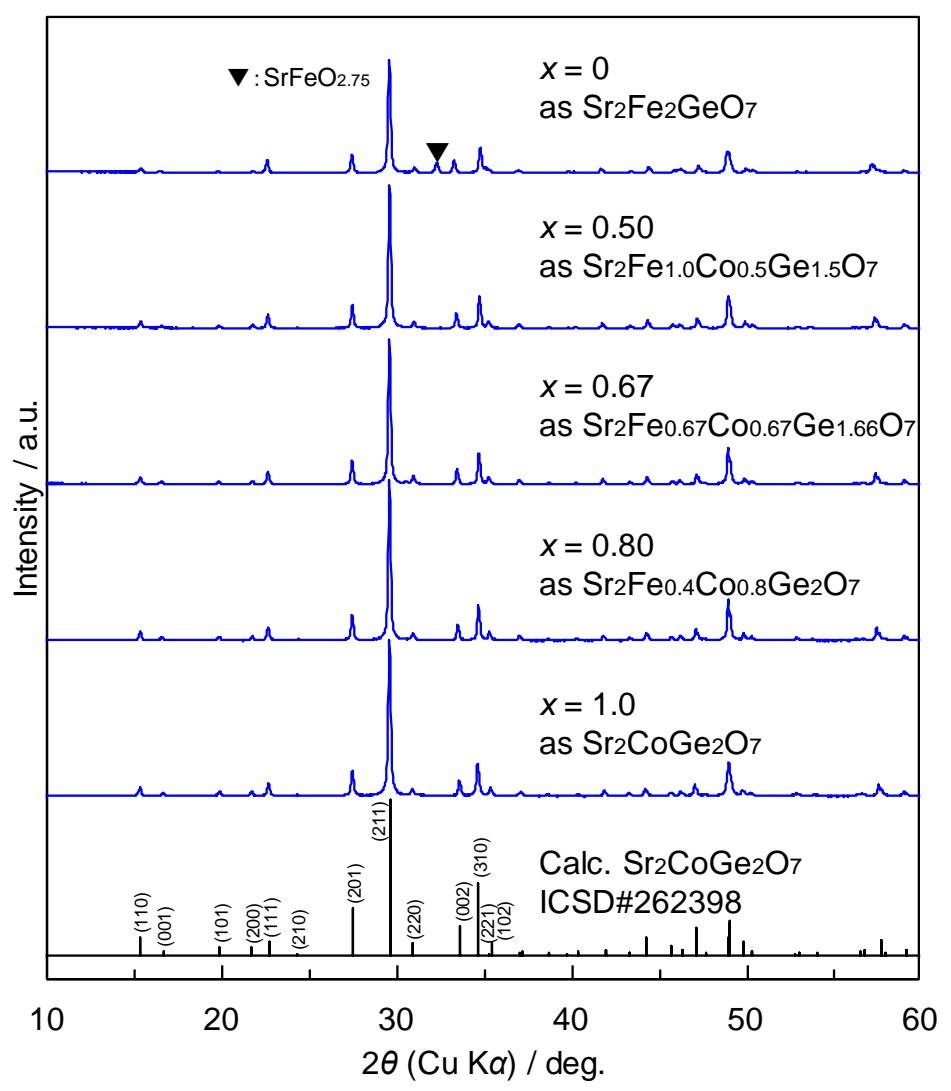

(a)

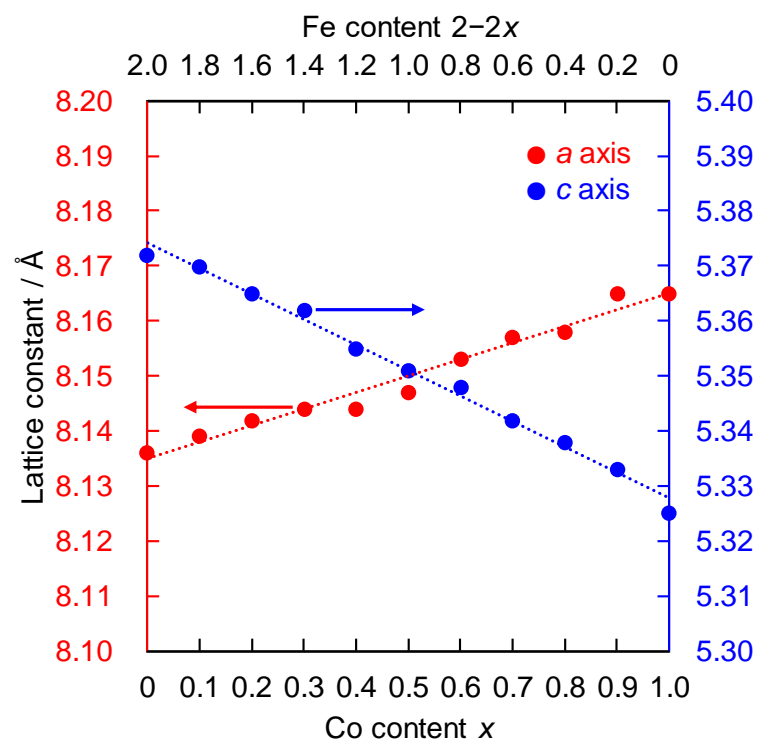

(b)

Table S1. (a) XRD patterns for $\mathrm{Sr}_{2} \mathrm{Fe}_{2}-2 x \mathrm{Co}_{x} \mathrm{Ge}_{1+x} \mathrm{O}_{7}(0 \leq x \leq 1)$. (b) The lattice constants $a$ and $c$ against $x$ in $\mathrm{Sr}_{2} \mathrm{Fe}_{2}-2 x \mathrm{Co}_{x} \mathrm{Ge}_{1+x} \mathrm{O}_{7}$. 


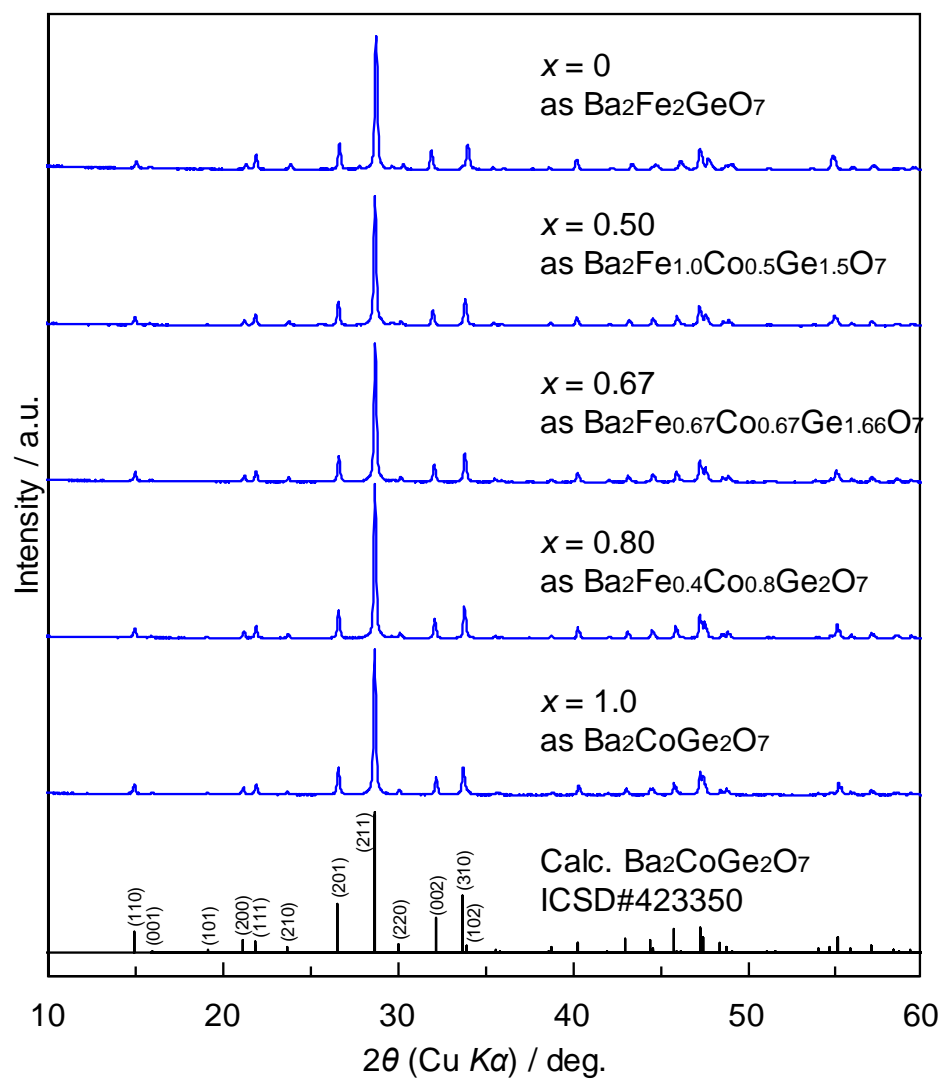

Figure S2. XRD patterns for $\mathrm{Ba}_{2} \mathrm{Fe}_{2}-2 x \mathrm{Co}_{x} \mathrm{Ge}_{1+x} \mathrm{O}_{7}(0 \leq x \leq 1)$. 

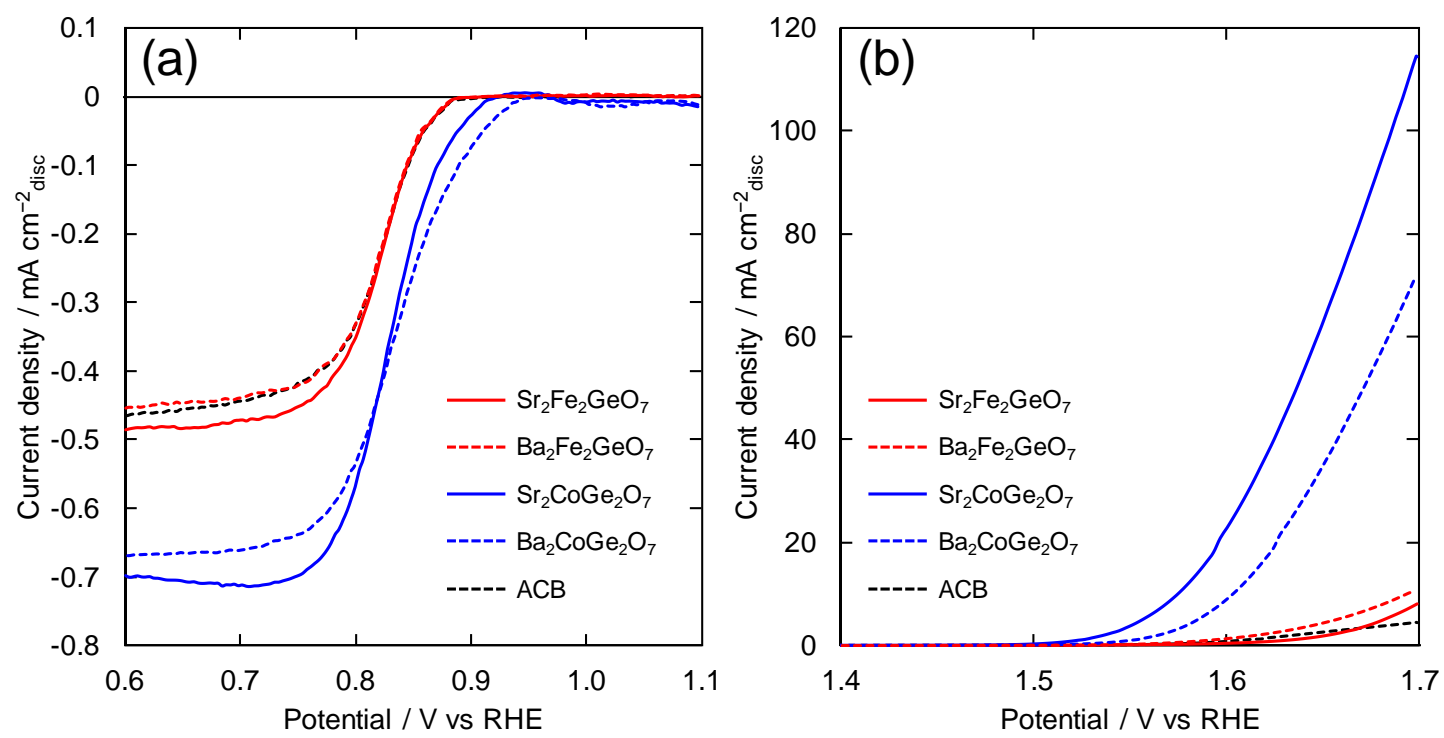

Figure S3. (a) ORR and (b) OER polarization curves of melilite-type iron and cobalt oxides. Solid and broken curves denote the data of the strontium and barium systems, respectively. 

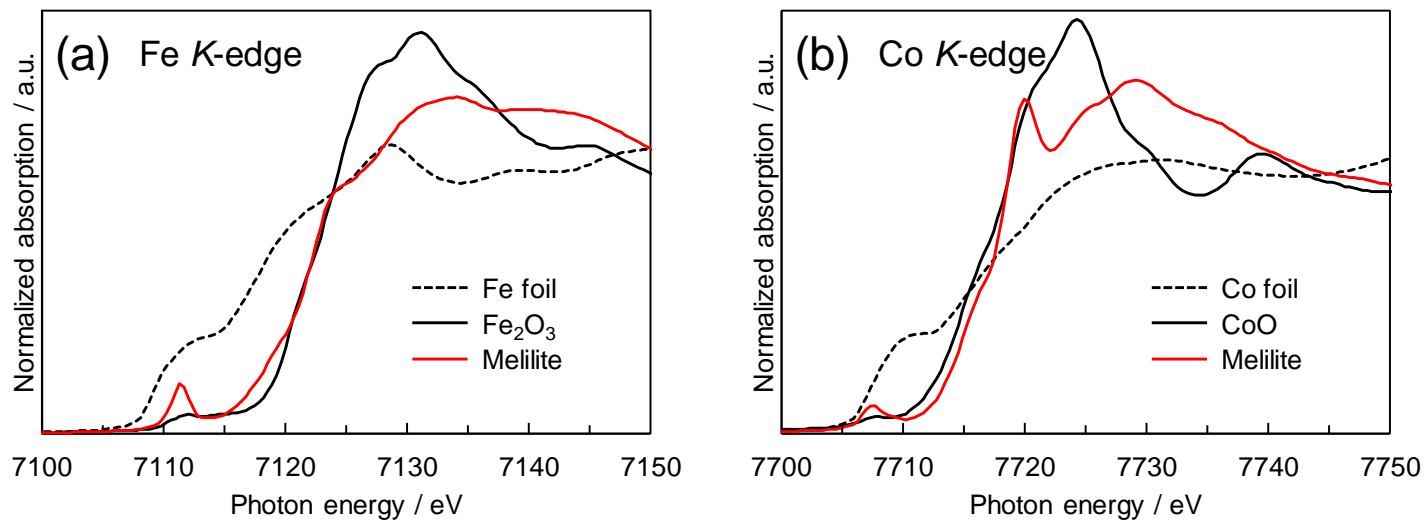

Figure S4. (a) $\mathrm{Fe} K$-edge and (b) Co $K$-edge XANES spectra of melilite-type $\mathrm{Sr}_{2} \mathrm{Fe}_{0.67} \mathrm{Co}_{0.67} \mathrm{Ge}_{1.66} \mathrm{O}_{7}$ together with those of the reference samples. 

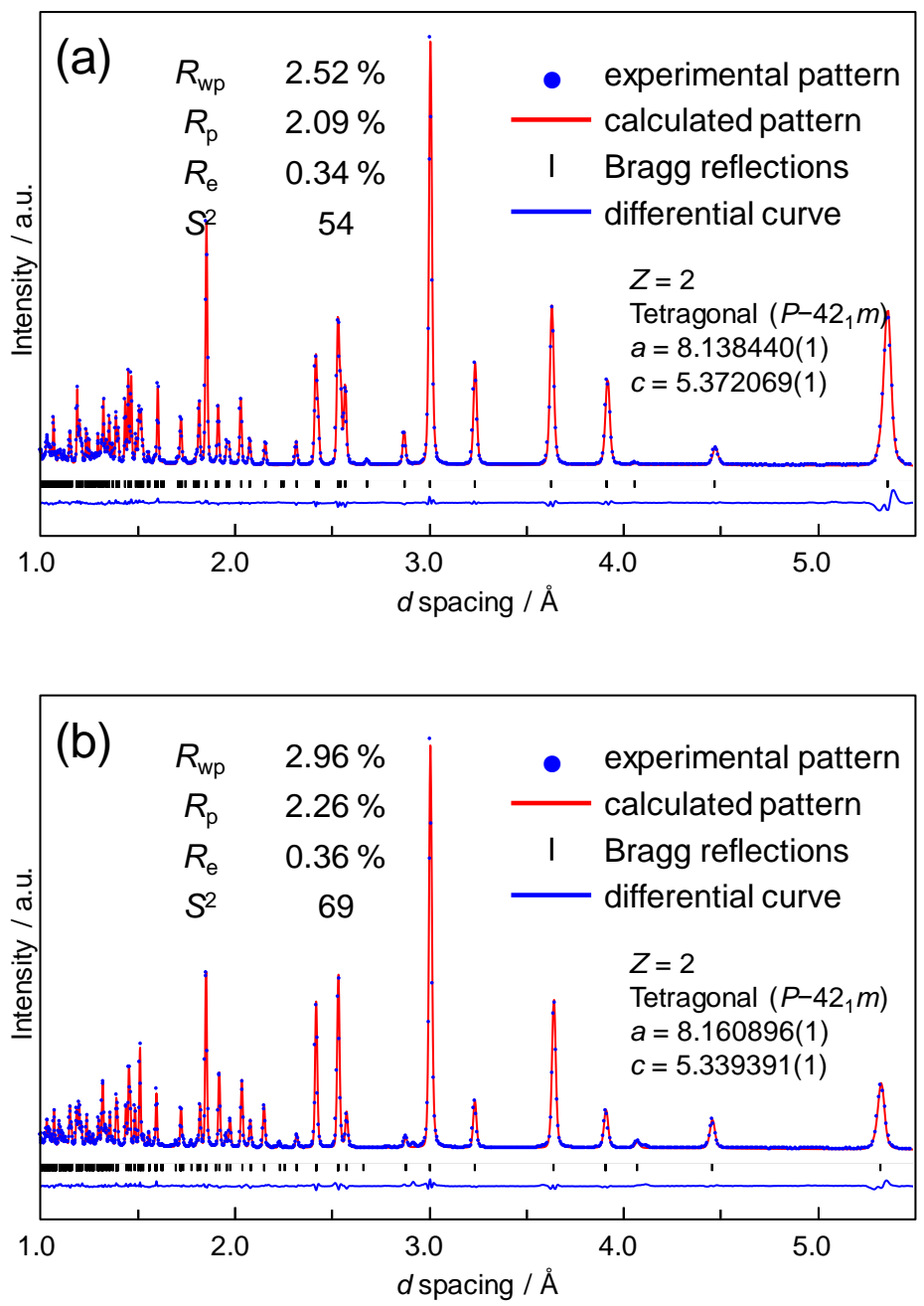

Figure S5. Neutron powder diffraction profiles of (a) $\mathrm{Sr}_{2} \mathrm{Fe}_{2} \mathrm{GeO}_{7}$ and (b) $\mathrm{Sr}_{2} \mathrm{Fe}_{0.4} \mathrm{Co}_{0 .} \mathrm{Ge}_{1.8} \mathrm{O}_{7}$. The calculated patterns by Rietveld refinement and the observed patterns are shown as the red curves and the blue dots, respectively. The vertical black marks in the middle show calculated Bragg reflection positions. The bottom blue curves are differences between the calculated and observed patterns. 
Table S1. Structural parameters of $\mathrm{Sr}_{2} \mathrm{Fe}_{2-2 x} \mathrm{Co}_{x} \mathrm{Ge}_{1+x} \mathrm{O}_{7}(x=0,0.67$ and 0.80$)$. $\mathrm{Sr}_{2} \mathrm{Fe}_{2} \mathrm{GeO}_{7}(x=0)$

\begin{tabular}{ccccccc}
\hline Atom & $x$ & $y$ & $z$ & $B_{\text {iso }} / \AA^{2}$ & Site & Sym. \\
\hline $\mathrm{Sr} 1$ & $0.16188(2)$ & 0.66188 & $0.50829(4)$ & $0.669(4)$ & $4 e$ &.. $\mathrm{~m}$ \\
$\mathrm{Fe} 1$ & 0 & 0 & 0 & $0.117(6)$ & $2 a$ & $-4 .$. \\
$\mathrm{Ge} 1$ & 0 & 0 & 0 & $0.117(6)$ & $2 a$ & $-4 .$. \\
$\mathrm{Ge} 2$ & $0.64385(1)$ & 0.14385 & $0.96511(3)$ & $0.122(4)$ & $4 e$ &.. $\mathrm{~m}$ \\
$\mathrm{Fe} 2$ & $0.64385(1)$ & 0.14385 & $0.96511(3)$ & $0.122(4)$ & $4 e$ &.. $\mathrm{~m}$ \\
$\mathrm{O} 1$ & 0 & 0.5 & $0.17931(7)$ & $0.662(7)$ & $2 c$ & $2 . \mathrm{mm}$ \\
$\mathrm{O} 2$ & $0.64032(2)$ & 0.14032 & $0.29314(4)$ & $0.635(5)$ & $4 e$ &.. $\mathrm{~m}$ \\
$\mathrm{O} 3$ & $0.08676(2)$ & $0.16707(2)$ & $0.19639(4)$ & $1.020(4)$ & $8 f$ & 1 \\
\hline
\end{tabular}

$\mathrm{Sr}_{2} \mathrm{Fe}_{0.67} \mathrm{Co}_{0.67} \mathrm{Ge}_{1.66} \mathrm{O}_{7}(x=0.67)$

\begin{tabular}{ccccccc}
\hline Atom & $x$ & $y$ & $z$ & $B_{\text {iso }} / \AA^{2}$ & Site & Sym. \\
\hline $\mathrm{Sr} 1$ & $0.16451(2)$ & 0.66451 & $0.50708(4)$ & $0.680(5)$ & $4 e$ &.. $\mathrm{~m}$ \\
$\mathrm{Co} 1$ & 0 & 0 & 0 & $0.163(10)$ & $2 a$ & $-4 .$. \\
$\mathrm{Fe} 1$ & 0 & 0 & 0 & $0.163(10)$ & $2 a$ & $-4 .$. \\
$\mathrm{Ge} 1$ & 0 & 0 & 0 & $0.163(10)$ & $2 a$ & $-4 .$. \\
$\mathrm{Ge} 2$ & $0.64325(2)$ & 0.14325 & $0.95648(3)$ & $0.222(4)$ & $4 e$ &.. $\mathrm{~m}$ \\
$\mathrm{Fe} 2$ & $0.64325(2)$ & 0.14325 & $0.95648(3)$ & $0.222(4)$ & $4 e$ &.. $\mathrm{~m}$ \\
$\mathrm{Co} 2$ & $0.64325(2)$ & 0.14325 & $0.95648(3)$ & $0.222(4)$ & $4 e$ &.. $\mathrm{~m}$ \\
$\mathrm{O} 1$ & 0 & 0.5 & $0.18061(7)$ & $0.782(8)$ & $2 c$ & $2 . \mathrm{mm}$ \\
$\mathrm{O} 2$ & $0.63973(2)$ & 0.13973 & $0.28059(5)$ & $0.710(5)$ & $4 e$ &.. $\mathrm{~m}$ \\
$\mathrm{O} 3$ & $0.08429(2)$ & $0.17446(2)$ & $0.20291(4)$ & $1.057(4)$ & $8 f$ & 1 \\
\hline
\end{tabular}

$\mathrm{Sr}_{2} \mathrm{Fe}_{0.4} \mathrm{Co}_{0.8} \mathrm{Ge}_{1.8} \mathrm{O}_{7}(x=0.80)$

\begin{tabular}{ccccccc}
\hline Atom & $x$ & $y$ & $z$ & $B_{\text {iso }} / \AA^{2}$ & Site & Sym. \\
\hline Sr1 & $0.16494(2)$ & 0.66494 & $0.50669(5)$ & $0.690(5)$ & $4 e$ &.. $\mathrm{~m}$ \\
$\mathrm{Co} 1$ & 0 & 0 & 0 & $0.142(13)$ & $2 a$ & $-4 .$. \\
$\mathrm{Fe} 1$ & 0 & 0 & 0 & $0.142(13)$ & $2 a$ & $-4 .$. \\
$\mathrm{Ge} 1$ & 0 & 0 & 0 & $0.142(13)$ & $2 a$ & $-4 .$. \\
$\mathrm{Ge} 2$ & $0.64317(2)$ & 0.14317 & $0.95471(4)$ & $0.238(4)$ & $4 e$ &.. $\mathrm{~m}$ \\
$\mathrm{Fe} 2$ & $0.64317(2)$ & 0.14317 & $0.95471(4)$ & $0.238(4)$ & $4 e$ &.. $\mathrm{~m}$ \\
$\mathrm{Co} 2$ & $0.64317(2)$ & 0.14317 & $0.95471(4)$ & $0.238(4)$ & $4 e$ &.. $\mathrm{~m}$ \\
$\mathrm{O} 1$ & 0 & 0.5 & $0.18070(8)$ & $0.711(8)$ & $2 c$ & $2 . \mathrm{mm}$ \\
$\mathrm{O} 2$ & $0.63970(2)$ & 0.13970 & $0.27767(5)$ & $0.678(6)$ & $4 e$ &.. $\mathrm{~m}$ \\
$\mathrm{O} 3$ & $0.08372(2)$ & $0.17624(2)$ & $0.20441(4)$ & $1.000(5)$ & $8 f$ & 1 \\
\hline
\end{tabular}




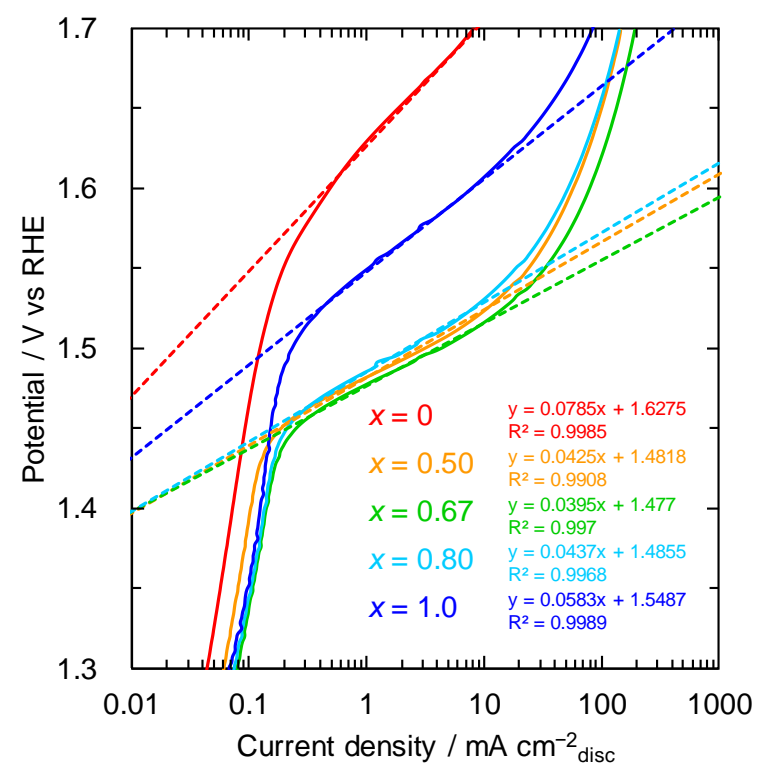

Figure S6. Tafel plots for the OER data of melilite-type $\mathrm{Sr}_{2} \mathrm{Fe}_{2}-2 x \mathrm{Co}_{x} \mathrm{Ge}_{1+}{ }_{1} \mathrm{O}_{7}$. 
Table S2. BET specific surface areas of melilite-type $\mathrm{Sr}_{2} \mathrm{Fe}_{2-2 x} \mathrm{Co}_{x} \mathrm{Ge}_{1+x} \mathrm{O}_{7}$. The value of the reference catalyst $\mathrm{RuO}_{2}$ is also shown.

\begin{tabular}{ccc}
\hline Sample & BET surface area $/ \mathrm{m}^{2} \mathrm{~g}^{-1}$ \\
\hline $\mathrm{Sr}_{2} \mathrm{CoGe}_{2} \mathrm{O}_{7}$ & $(x=1.0)$ & 2.67 \\
$\mathrm{Sr}_{2} \mathrm{Fe}_{0.4} \mathrm{Co}_{0.8} \mathrm{Ge}_{1.8} \mathrm{O}_{7}$ & $(x=0.80)$ & 1.28 \\
$\mathrm{Sr}_{2} \mathrm{Fe}_{0.67} \mathrm{Co}_{0.677} \mathrm{Ge}_{1.66} \mathrm{O}_{7}$ & $(x=0.67)$ & 1.92 \\
$\mathrm{Sr}_{2} \mathrm{FeCo}_{0.5} \mathrm{Ge}_{1.5} \mathrm{O}_{7}$ & $(x=0.50)$ & 1.86 \\
$\mathrm{Sr}_{2} \mathrm{Fe}_{2.0} \mathrm{GeO}_{7}$ & $(x=0)$ & 1.70 \\
$\mathrm{Ba}_{2} \mathrm{CoGe}_{2} \mathrm{O}_{7}$ & $(x=1.0)$ & 1.26 \\
$\mathrm{RuO}_{2}$ & & 10.3
\end{tabular}




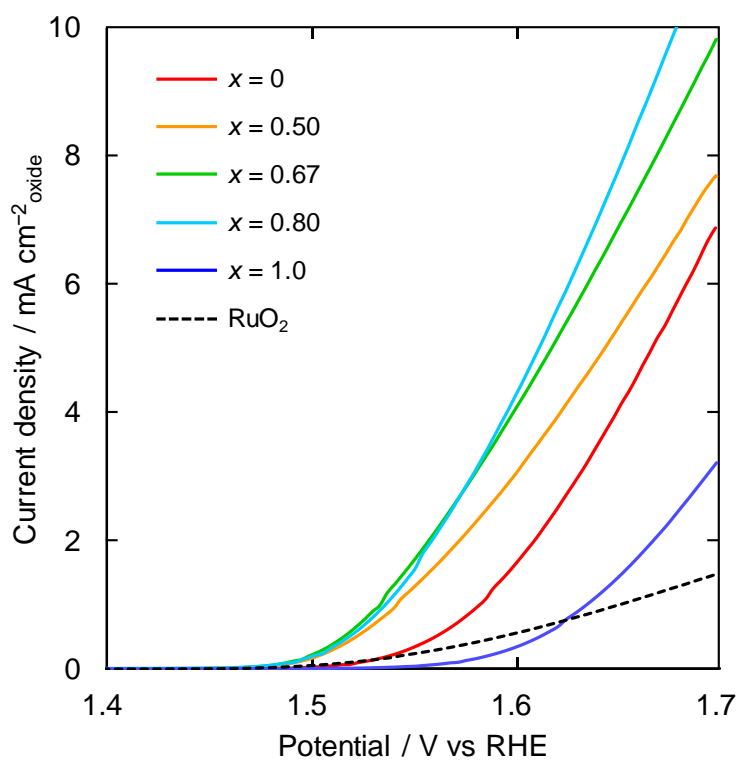

Figure S7. OER polarization curves of melilite-type $\mathrm{Sr}_{2} \mathrm{Fe}_{2}-2 x \mathrm{Co}_{x} \mathrm{Ge}_{1+x} \mathrm{O}_{7}$ together with that of the reference catalyst $\mathrm{RuO}_{2}$. The current density values are normalized by the specific surface area of the catalyst. 
Synthesis of Fe/Co-mixed oxides of various structural types. Oxygen-deficient perovskite $\mathrm{Ba} 0.5 \mathrm{Sr}_{0.5} \mathrm{Co}_{0.8} \mathrm{Fe}_{0.2} \mathrm{O}_{3}-\delta$, garnet-type $\mathrm{Y}_{3} \mathrm{Fe}_{2} \mathrm{Co}_{1.5} \mathrm{Ge}_{1.5} \mathrm{O}_{12}$, and spinel-type $\mathrm{Fe}_{1.5} \mathrm{Co} 1.5 \mathrm{O}_{4}$ were synthesized via a citrate precursor route, as described below. $\mathrm{Sr}\left(\mathrm{NO}_{3}\right)_{2}$ (99.5\%, Kanto Chemical), $\mathrm{BaCO}_{3}$ (99.9\%, FUJIFILM Wako Pure Chemical), $\mathrm{Y}_{2} \mathrm{O}_{3}$ (99.9\%, Kojundo Chemical Laboratory), $\mathrm{Fe}\left(\mathrm{NO}_{3}\right)_{3} \cdot 9 \mathrm{H}_{2} \mathrm{O}$ (99.9\%, Kanto Chemical), $\mathrm{Co}\left(\mathrm{NO}_{3}\right)_{2} \cdot 6 \mathrm{H}_{2} \mathrm{O}$ (99.9\%, Kojundo Chemical Laboratory), $\mathrm{GeO}_{2}$ (99.99\%, Kojundo Chemical Laboratory), lactic acid (FUJIFILM Wako Pure Chemical), nitric acid (80\%, FUJIFILM Wako Pure Chemical) and citric acid (98.0\%, FUJIFILM Wako Pure Chemical) were used as starting materials. Water-insoluble $\mathrm{BaCO}_{3}$ and $\mathrm{Y}_{2} \mathrm{O}_{3}$ were separately dissolved in lactic-acid and nitric-acid aqueous solutions, respectively. Appropriate amounts of these reagents were dissolved in a citric acid aqueous solution. The molar ratio of metal ions to citric acid was $1 / 3$. The citrate solution was stirred and heated at $120{ }^{\circ} \mathrm{C}$ overnight to obtain a homogeneous gel. The gelatinous product was calcined at $450{ }^{\circ} \mathrm{C}$ for 3 hours in a mantle heater, followed by grinding and firing at 1000 ${ }^{\circ} \mathrm{C}$ in air for 10 hours. 


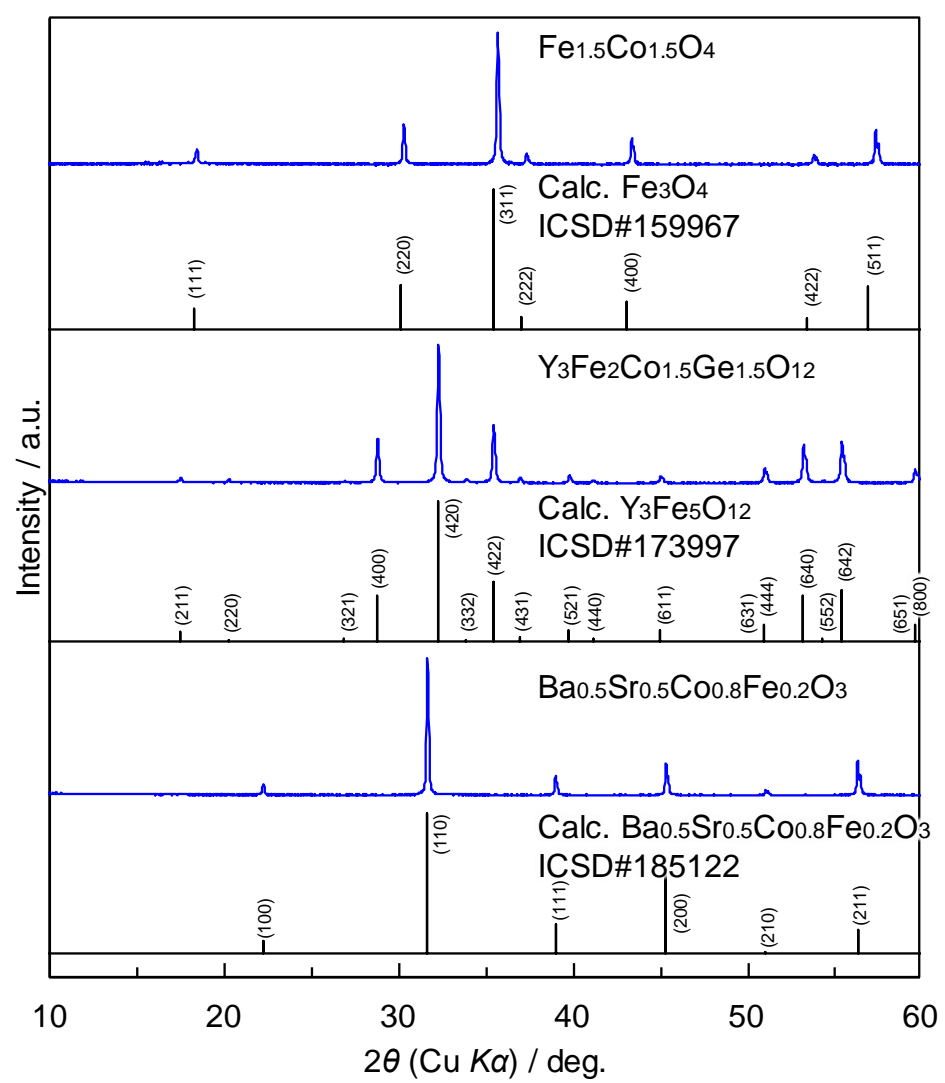

Figure S8. XRD patterns for the Fe/Co-mixed oxides of various structural types: oxygendeficient perovskite $\mathrm{Ba} 0.5 \mathrm{Sr}_{0.5} \mathrm{Co}_{0.8} \mathrm{Fe}_{0.2} \mathrm{O}_{3}-\delta$, garnet-type $\mathrm{Y}_{3} \mathrm{Fe}_{2} \mathrm{Co}_{1.5} \mathrm{Ge}_{1.5} \mathrm{O}_{12}$, and spineltype $\mathrm{Fe}_{1.5} \mathrm{Co}_{1.5} \mathrm{O}_{4}$.

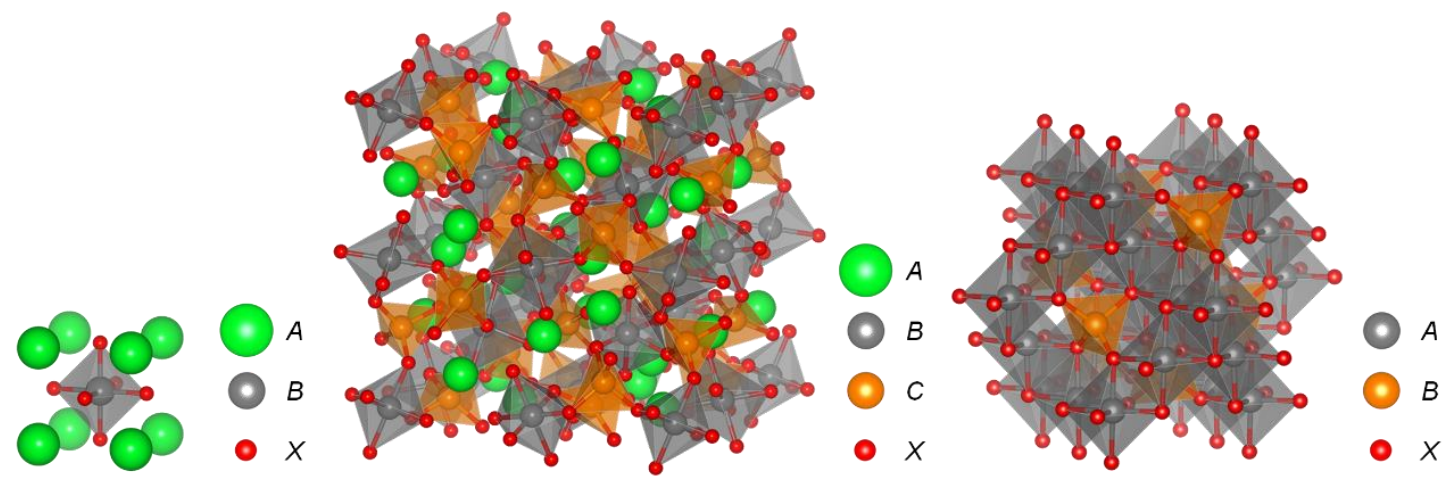

(a) $A B X_{3}$

(b) $A_{3} B_{2} C_{3} X_{12}$

(c) $A B_{2} X_{4}$

Figure S9. Crystal structures of (a) perovskite-type, (b) garnet-type, and (c) spinel-type compounds. The grayish and brownish polyhedra denote octahedrally- and tetrahedrallycoordinated sites, respectively. 
Table S3. Specific surface areas of the Fe/Co-mixed oxides of various structural types.

\begin{tabular}{cc}
\hline Composition & BET surface area $/ \mathrm{m}^{2} \mathrm{~g}^{-1}$ \\
\hline $\mathrm{Fe}_{1.5} \mathrm{Co}_{1.5} \mathrm{O}_{4}$ & 2.27 \\
$\mathrm{Y}_{3} \mathrm{Fe}_{2} \mathrm{Co}_{1.5} \mathrm{Ge}_{1.5} \mathrm{O}_{12}$ & 5.38 \\
$\mathrm{Ba}_{0.5} \mathrm{Sr}_{0.5} \mathrm{Co}_{0.8} \mathrm{Fe}_{0.2} \mathrm{O}_{3}$ & 2.46 \\
\hline
\end{tabular}

Table S4. Chemical composition ratios of the $\mathrm{Sr}_{2} \mathrm{Fe}_{0.67} \mathrm{Co}_{0.67} \mathrm{Ge}_{1.66} \mathrm{O}_{7}$ catalysts determined by EDX analysis.

\begin{tabular}{cccc}
\hline Element & $\begin{array}{c}\text { Atomic percent } \\
\text { (Ideal) } / \%\end{array}$ & $\begin{array}{c}\text { Atomic percent } \\
\text { (as-prepared) } / \%\end{array}$ & $\begin{array}{c}\text { Atomic percent } \\
\text { (after ECR, } \\
\text { surface) } / \%\end{array}$ \\
\hline $\mathrm{Sr}$ & 40.0 & 40.4 & 10.4 \\
$\mathrm{Fe}$ & 13.4 & 14.1 & 37.3 \\
$\mathrm{Co}$ & 13.4 & 14.0 & 44.8 \\
$\mathrm{Ge}$ & 33.2 & 31.5 & 7.5 \\
\hline
\end{tabular}


Durability tests for the $\mathrm{Sr}_{2} \mathrm{Fe}_{0.67} \mathrm{Co0}_{0.67} \mathrm{Ge}_{1.66} \mathrm{O}_{7}$ catalyst in highly alkaline media. A portion of the as-synthesized $\mathrm{Sr}_{2} \mathrm{Fe}_{0.67} \mathrm{Co}_{0.677} \mathrm{Ge}_{1.66} \mathrm{O}_{7}$ sample $(0.2 \mathrm{mg})$ was immersed in a $4 \mathrm{M} \mathrm{KOH}$ aqueous solution while stirring at $50{ }^{\circ} \mathrm{C}$ for 72 hours. For electrochemical durability tests, working electrodes were prepared as follows. A catalyst slurry was similarly prepared as described in the main text. A gold foil $(10 \mathrm{~mm} \times 40 \mathrm{~mm} \times 10 \mu \mathrm{m})$ was used as a current collector. $1.0 \mathrm{~mL}$ of the slurry was cast onto the gold foil within a $10 \times 10 \mathrm{~mm}$ square. Then, chronoamperometry at $1.70 \mathrm{~V}$ vs RHE and chronopotentiometry at $10 \mathrm{~mA} / \mathrm{cm}^{2}$ were performed for 6 and 12 hours, respectively. These measurements were conducted in a $4 \mathrm{M} \mathrm{KOH}$ aqueous solution at $25{ }^{\circ} \mathrm{C}$.

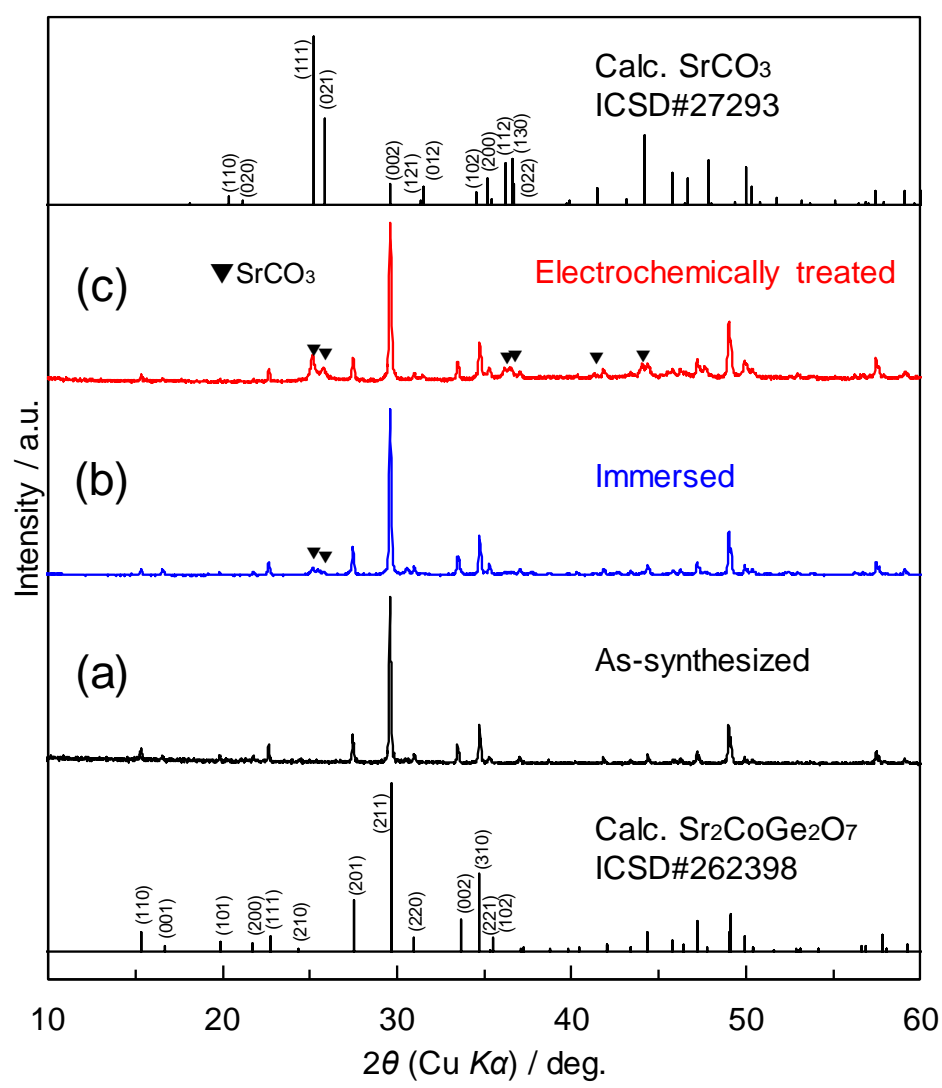

Figure S10. XRD patterns for (a) as-prepared $\mathrm{Sr}_{2} \mathrm{Fe}_{0.67} \mathrm{Co}_{0.67} \mathrm{Ge}_{1.66} \mathrm{O}_{7}$, (b) the sample immersed in a $4 \mathrm{M} \mathrm{KOH}$ aqueous solution at $50{ }^{\circ} \mathrm{C}$ for 72 hours and (c) the sample electrochemically-treated at $1.70 \mathrm{~V}$ vs RHE for 6 hours in a $4 \mathrm{M} \mathrm{KOH}$ aqueous solution. 


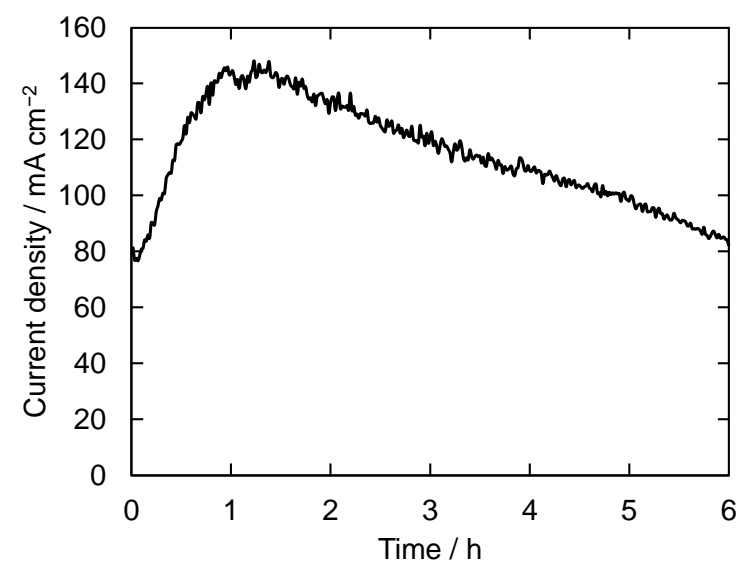

Figure S11. Chronoamperometric curve of the $\mathrm{Sr}_{2} \mathrm{Fe}_{0.67} \mathrm{Co}_{0.67} \mathrm{Ge}_{1.66} \mathrm{O}_{7}$ catalyst with an applied potential of $1.70 \mathrm{~V}$ vs RHE for 6 hours in a $4 \mathrm{M} \mathrm{KOH}$ aqueous solution.

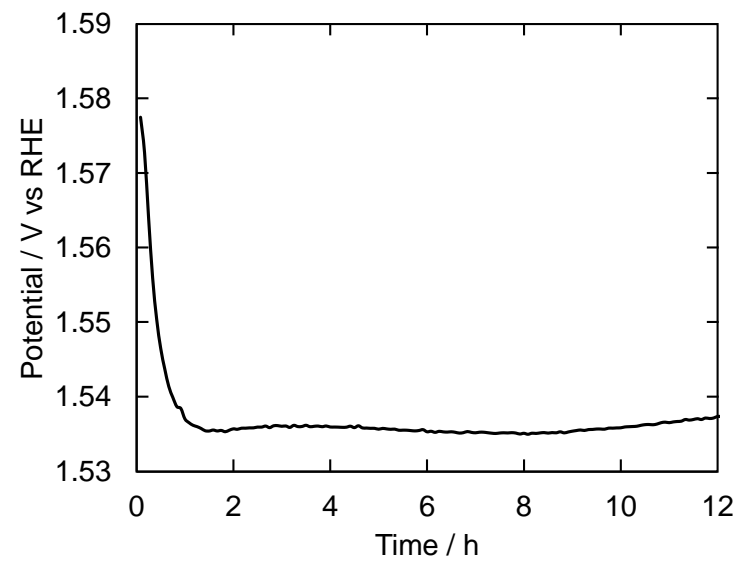

Figure S12. Chronopotentiometric curve of the $\mathrm{Sr}_{2} \mathrm{Fe}_{0.67} \mathrm{Co}_{0.67} \mathrm{Ge}_{1.66} \mathrm{O}_{7}$ catalyst with an applied current density of $10 \mathrm{~mA} / \mathrm{cm}^{2}$ for 12 hours in a $4 \mathrm{M} \mathrm{KOH}$ aqueous solution. 